\title{
Chapter 9 \\ Chromosomal Changes over the Course of Polyploid Wheat Evolution and Domestication
}

\author{
Ekaterina D. Badaeva, Olga S. Dedkova, V.A. Pukhalskyi, and A.V. Zelenin
}

\begin{abstract}
Polyploid wheats are represented by two evolutionary lineages - Emmer and Timopheevi. It was reported that the species of these groups differentiated by species-specific translocations; they showed distinct karyotype structures, i.e., the amount and the distribution of heterochromatin. Analysis of more than 1,500 accessions representing 21 wild and cultivated polyploid wheat species using C-banding revealed that intra- and interspecific divergence within these two groups was accompanied by chromosomal rearrangements. Intraspecific diversity was the highest for wild species, followed by landraces and commercial cultivars. Chromosomal rearrangements were more frequent in T. araraticum than in $T$. dicoccoides $(55.7 \%$ and $35.3 \%$ correspondingly). Altogether, 2 pericentric inversions, 28 single translocations, 13 double translocations, and five multiple translocations were identified in 150 of 270 T. araraticum accessions. Sixty types of chromosomal rearrangements (4 inversions, 37 single translocations, 11 double and 6 triple translocations, and two unclassified rearrangements) were found in T. dicoccoides (143 of 400 accessions). The range of karyotype diversity decreased in cultivated Emmer: 25 single translocations, 4 pericentric inversions, 6 double and 3 multiple translocations were detected in 119 of 470 accessions (24.5\%). The translocation T5B:7A significantly dominated over other variants; alone or in combination with other translocations, being identified in 51 lines of $T$. dicoccum (Schrank) Schübler, preferentially from Western Europe and Mediterranean countries. Chromosomal rearrangements were also found in common wheat, the translocations T5B:7B and wheat-rye T1RS:1BL being the most frequent ( 25 and 29 cultivars respectively). In addition to them, 24 variants of chromosomal rearrangements, including inversions, single and multiple translocations and wheat-alien translocations and substitutions were discovered in 112 of 295 cultivars we studied.
\end{abstract}

\footnotetext{
E.D. Badaeva $(\varangle) \cdot$ A.V. Zelenin

Engelhardt Institute of Molecular Biology, Russian Academy of Sciences, Vavilov street, 32, Moscow 119991, Russia

e-mail: katerinabadaeva@gmail.com

O.S. Dedkova $\bullet$ V.A. Pukhalskyi

N.I. Vavilov Institute of General Genetics, Russian Academy of Sciences,

Gubkin street, 3, Moscow 119991, Russia
} 
Keywords C-banding $\bullet$ Chromosomes $\bullet$ Domestication • Evolution $\bullet$ Inversions $\bullet$ Polymorphism • Translocations $\bullet$ Triticum

Wheat evolution and domestication have been attracting the attention of researchers for over a 100 years, and a broad range of methods have been applied to uncover their genetic mechanisms. These studies have revealed that polyploid wheats are represented by two evolutionarily distinct groups: Emmer and Timopheevi that originated independently from hybridization of two diploid species: Aegilops speltoides Tausch. as a female parent and Triticum urartu Tum. ex. Gandil. as a male parent (Dvorák 1998). Hexaploid bread wheat arose from hybridization of an unknown tetraploid wheat species with Aegilops tauschii Coss. (Kihara 1944).

Examination of meiotic chromosome pairing in $\mathrm{F}_{1}$ hybrid of T. turgidum L. x T. timopheevii Zhuk., experiments with Genomic in situ Hybridization (GISH) and the construction of high-density molecular and genetic maps of common wheat and $T$. timopheevii showed that the emergence of polyploid wheats was accompanied by species-specific chromosomal rearrangements: translocation 4A-5A-7B with subsequent pericentric inversion in the chromosome $4 \mathrm{~A}$ in Emmer group and 1G-4G$6 A^{t}+3 A^{t}-4 A^{t}$ in Timopheevi group (Jiang and Gill 1994; Liu et al. 1992; Naranjo et al. 1987; Rodríguez et al. 2000; Salina et al. 2006). Further analyses of intraspecific and interspecific diversity in various wheat species revealed differences between species as well as between geographic populations of the same species with respect to nuclear and cytoplasmic genome polymorphisms (Kilian et al. 2007; Mori et al. 2009; Salamini et al. 2002), isozyme and storage protein spectra (Belay 2000; Nishikawa 1984), and C-banding patterns and chromosomal rearrangements (Badaeva et al. 1994; Dedkova et al. 2009; Kawahara 1997; Kawahara and Taketa 2000; Taketa and Kawahara 1996). These studies however included a limited number of species, which does not allow obtaining a general overview of the wheat genome evolution. In the present work we analyzed all Triticum species belonging to Emmer and Timopheevi groups using C-banding method to discover karyotype alterations associated with evolution and domestication of polyploid wheat.

Our chromosome analysis demonstrates that intra- and interspecific divergence within Emmer and Timopheevi groups is accompanied by chromosomal rearrangements. Intraspecific diversity is the highest in wild species; and the two evolutionary groups display different mechanisms of karyotype evolution. Chromosomal rearrangements are more frequent in T. araraticum Jakubz. than in $T$. dicoccoides (Körn. ex Aschers. \& Graebn.) Schweinf. (55.7 \% and $35.3 \%$, lines, respectively). Translocation occur preferentially via the centromeric-breakagefusion mechanism ( 63 centromeric vs. 9 interstitial breakage events led to the emergence of totally 28 types of single and 18 complex translocations), whereas the ratio between centromeric/interstitial translocation in wild Emmer is not so extreme (48 centromeric vs. 34 interstitial translocation events). In both lineages translocations among $\mathrm{G}$ and $\mathrm{B}$ genome chromosomes are the most frequent (66 and 82 variants of rearranged chromosomes are formed by B-B or G-G arm combinations respec-

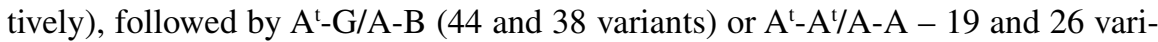
ants of rearranged chromosomes, respectively). 
Altogether, 48 different variants of chromosomal rearrangements, including 2 pericentric inversions, 28 single translocations, 13 double translocations and 5 multiple translocations, are identified in T. araraticum (150 of 270 accessions), and among them 13 translocations are novel. Most rearrangements (24 variants) are found in each of the single accessions, whereas 3 types occur more frequently. One of them is pericentric inversion in chromosome $7 \mathrm{~A}^{\mathrm{t}}$ which is present in 15 Iranian and 3 Iraqi (Sulaimaniyah) accessions. The second is a translocation T6G:7G identified in 12 accessions from Salah-ad-Din (Iraq). The third, multiple translocation T4A $A^{t}: 7 A^{t}+T 2 A: 4 G: 7 G$ dominates in Nakhichevan, Azerbaijan (13 accessions), however, it has been never observed in other regions of the Caucasus. It might be occurred as a result of three subsequent translocations and, in turn, gave rise to another variant of multiple translocations found in Nakhichevan. Another translocation series was also observed in Armenia (Badaeva et al. 1994).

Triticum dicoccoides also shows high diversity in the C-banding patterns. Two distinct karyotypic groups are able to be distinguished in this species. The first group is typical for the subspecies judaicum, which is distributed north and west of the Galilee Sea. These accessions show characteristic C-banding patterns in chromosomes 2A, 4A, 7A, 1B, 3B, 4B, 7B; and, especially, in chromosome 5B (Fig. 9.1). The second group is much larger and includes accessions from all areas of the species distribution. They show an extremely high C-banding polymorphism, however, no distinct karyotypic variant is able to be discriminated among the geographical

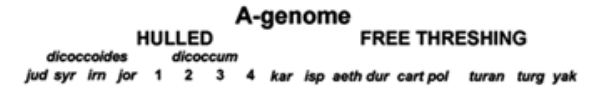

1

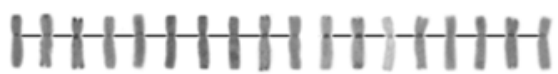

2
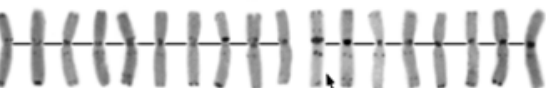

3
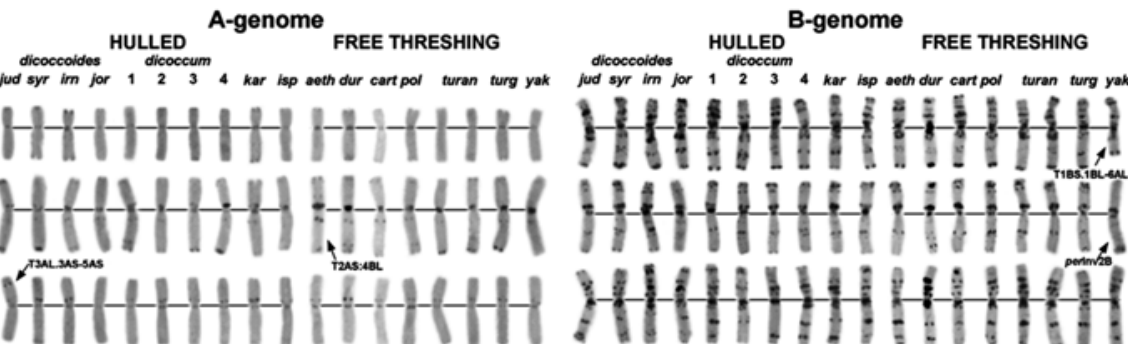

4

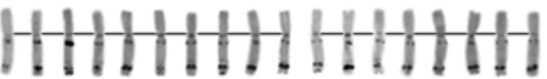

5
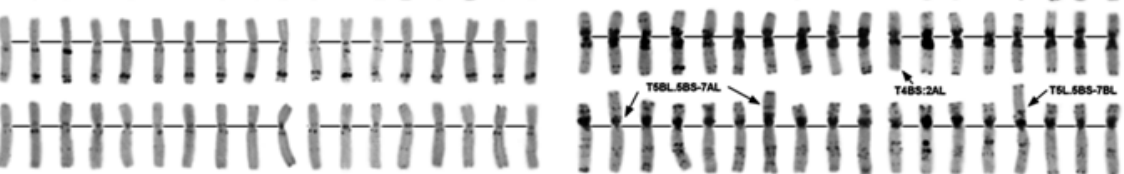

$6-8-1-8-1-1-1$
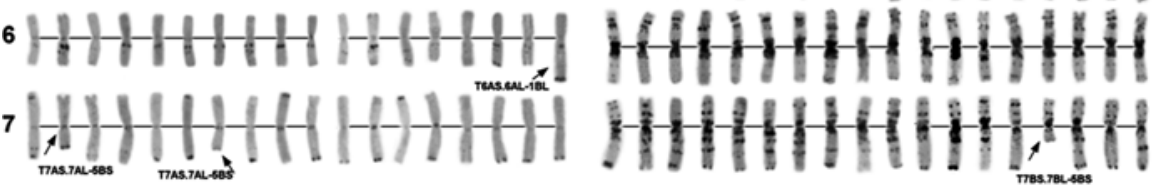

Fig. 9.1 Karyotype diversity in tetraploid wheat species of the Emmer group; dicoccoides $-T$. dicoccoides, jud - ssp. judaicum, syr - Syrian, irn - Iranian, jor - Jordanian accessions, dicoccum - T. dicoccum, 1 -Asian type, 2 - Balkan type, 3 - European type, 4 - Ethiopian type, $k a r-T$. karamyschevii Nevski, isp - T. ispahanicum Heslot, aeth - T. aethiopicum Jakubz., dur - T. durum Desf., cart - T. cathlicum Nevski, pol - T. polonicum L., turan - T. turanicum Jakubz., turg $-T$. turgidum, yak - T. yakubzinery Udacz. et Schachm. 1-7 - homoeologous groups. Chromosomal rearrangements are arrowed 
populations on the basis of C-banding analysis. The highest karyotype diversity is observed in Jordan Valley, Israel.

Sixty types of rearrangements (4 inversions, 37 single translocations, 11 double and 6 triple translocations, and 2 unclassified rearrangements) are identified in $T$. dicoccoides (143 of 400 accessions). Although most of them are detected in each of the single accessions (40 variants), some are found in two (6 variants), three (6 variants) or more (9 variants) accessions. If the same translocation is identified in several accessions, they are usually assumed to grow in the same geographic region. However, some translocation variants are exceptional. A translocation between 7A and $5 \mathrm{~B}$ chromosomes is identified in 5 accessions from Iraq, Syria, Lebanon, Israel and Armenia. Another, minor translocation between chromosomes $3 \mathrm{~A}$ and $5 \mathrm{~A}$ is found in 34 accessions of $T$. dicoccoides ssp. judaicum. In combination with other secondary translocations, three variants of multiple translocations are identified in five other accessions from Israel.

Karyotype diversity decreases in cultivated emmer compared to wild wheat; this is reflected by lower polymorphism in the $\mathrm{C}$-banding patterns, decreased frequencies and narrow spectra of chromosomal rearrangements. Altogether 25 single translocations, 4 pericentric inversions, 6 double and 3 multiple translocations are identified in 119 of 470 accessions we studied. Thirty four translocation types are observed in each of the single accessions, and nine are identified in three and more accessions; among them T6B:7B is found in the accessions from Montenegro, and two related translocations T1B:4A+T4B:6B+T1A:6A and T1B:4A+T4B:6B:T1A:6A - are found in those from Morocco. The most frequent translocation T5B:7A is identified in 45 accessions, and also in combination with secondary translocations, in other six accessions. It is found in 22 countries in Europe, Asia and Northern Africa, but is the most abundant in Western Europe and Mediterranean countries (25\% of the population). Although T5B:7A is detected in two T. dicoccum lines from Turkey, it is totally absent in Turkish population of wild emmer (63 accessions). As is mentioned above, a similar translocation has been identified in five Palestine accessions of $T$. dicoccoides. Based on this fact we assume that 5B:7A translocation was either transferred from wild to cultivated emmer owing to intercrossing of these species, or European emmer was independently domesticated from the local Palestine population of T. dicoccoides.

Comparison of tetraploid species of the Emmer group does not reveal any significant differences among them in karyotype structures and C-banding patterns (Fig. 9.1). Species with broad distribution are more polymorphic compared to the endemic species (T. karamyschevii, T. ispahanicum, T. turanicum, T. carthlicum, T. aethiopicum). The species T. yakubzinery is represented by a single accession, which carries a 1B:6A translocation and pericentric inversion in the chromosome 2B (Fig. 9.1). A translocation between chromosomes $2 \mathrm{~A}$ and $4 \mathrm{~B}$ is common in T. aethiopicum and is observed in $70 \%$ (present study) up to $100 \%$ of the accessions (Kawahara and Taketa 2000). Two of six T. turanicum accessions we studied (TRI 4326 and TRI 11533), one of 400 T. dicoccoides accessions (IG 117890, Syria), and one of $470 \mathrm{~T}$. dicoccum accessions (PI 308879, Spain) possess a 5B:7B translocation, which is very similar to the most widespread translocation of the European bread wheat in the structure of rearranged chromosomes. 

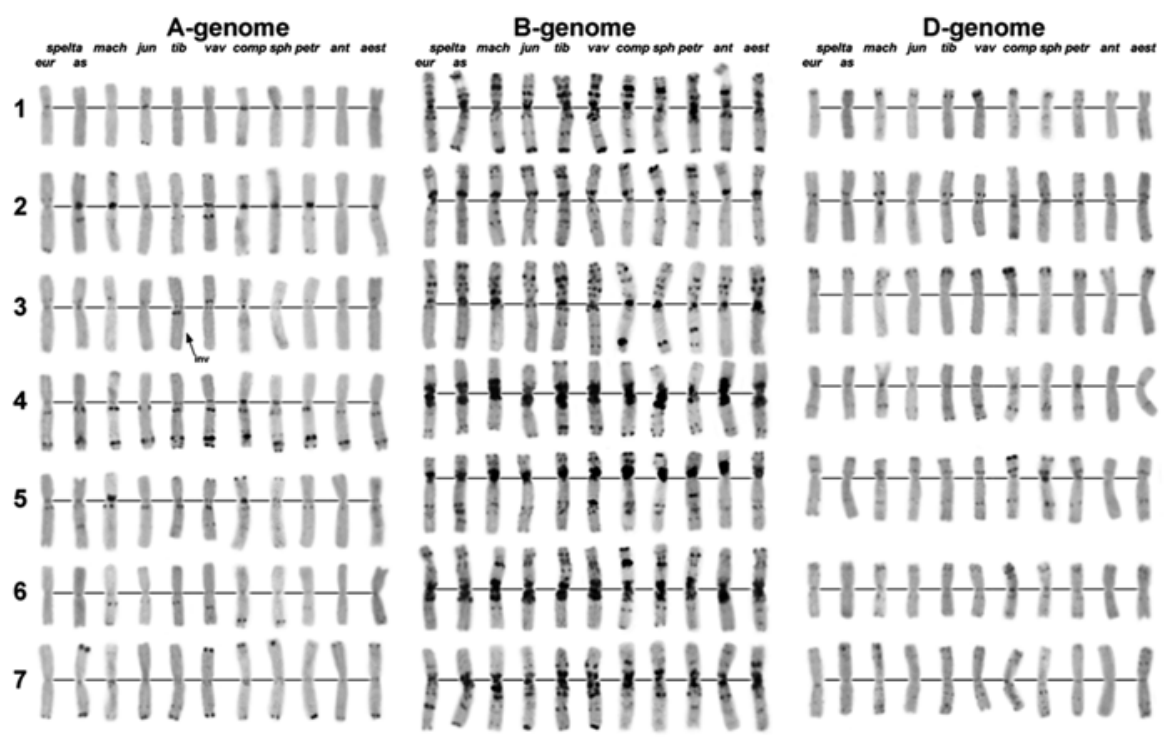

Fig. 9.2 Karyotype diversity in hexaploid wheat species of the Emmer group; splt- T. spelta L., eur - European type, as - Asian type, mach - T. macha Dekapr. et Menabde, jun - T. aesticum ssp. junnanense, tib - T. aestivum ssp. tibetianum Shao, vav - T. vavilovii (Thum.) Jakubz., comp - T. compactum Host, sph - T. sphaerococcum Perciv., petr - T. petropavlovskyi Udacz. et Migush., ant $-T$. antiquorum Heer ex Udacz, aest $-T$. aestivum L. em Thell. 1-7-homoeologous groups. Chromosomal rearrangements are arrowed

Hexaploid wheat species are similar to each other in the C-banding patterns of the A, B and D genome chromosomes (Fig. 9.2). Three species - bread wheat (T. aestivum), spelt (T. spelta) and club wheat (T. compactum) are highly polymorphic, whereas T. macha, T. vavilovii, T. sphaerococcum, T. petropavlovskyi, and two subspecies of common wheat $T$. aestivum ssp. junnanense and ssp. tibetianum show limited polymorphism; all these wheats possess characteristic banding patterns allowing discrimination among them. Two of the two T. aestivum ssp. tibetianum accessions we studied here carry the same modification of the chromosome 3A (Fig. 9.2). C-banding analysis reveals clear differences between European and Asian groups of spelt wheat; the C-band distribution on the chromosome 2A of European spelt is similar to that of the European Emmer, which further confirms that European spelt was a secondary crop that originated from hybridization of common wheat with $T$. dicoccum. The most frequent translocation in spelt is $\mathrm{T} 4 \mathrm{~A}: 1 \mathrm{~B}$, which is found in 8 accessions from Europe and Iran.

Polymorphism level decreases in common wheat compared to that in spelt, commercial varieties are less polymorphic than local varieties and landraces. Thirty five variants of chromosomal rearrangements and/or wheat-alien introgression are identified in 112 out of 295 common wheat cultivars. Translocation T5B:7B is the most widespread, being found in 25 cultivars studied and in at least 16 other cultivars described in literature (Friebe and Gill 1994; Schlegel 1996). 
Other rearrangements have much lower frequencies. Many cultivars and breeding lines of T. aestivum have been reported to carry wheat-alien substitutions and/or translocations (Friebe et al. 1996; Schneider et al. 2008). The wheat-rye translocation T1B:1R is the most frequent one followed by T1A:1R. Wheat-Agropyron, wheat-T. timopheevii, and wheat-Aegilops substitutions and translocations are also identified in several Russian wheat cultivars and breeding lines, most of them being associated with pest resistance.

Our study shows that the evolution and domestication of polyploid wheat is associated with karyotypic changes due to C-banding polymorphisms and chromosomal rearrangements. Variation of Giemsa banding patterns is highest in wild species. Geographic populations within T. araraticum and T. dicoccoides diverged as a result of emergence of region-specific polymorphic Giemsa bands. Domestication of wheat led to a significant decrease of C-banding polymorphisms. Among cultivated wheats endemic species show the lowest variation of the C-banding patterns which can be due to geographic isolation. Our results revealed an important role of chromosomal rearrangements in interspecific and intraspecific divergence of polyploid wheats. The spectra and frequencies of chromosomal rearrangements are species-specific and geographical region specific and can be used as cytogenetic markers in phylogenetic and evolutionary studies of wheat. On the other hand, broad occurrence of some chromosomal rearrangements can be an indicative of their adaptive significance.

Acknowledgments We thank Drs. O.P. Mitrofanova (VIR, St-Petersburg) and N.P. Goncharov (Institute of Cytology and Genetics, Novosibirsk, Russia); Dr. H. Bockelman (USDA-ARS, Aberdeen, Idaho) and Dr. B.S. Gill (Wheat Genetics and Genomics Resource Centre, Kansas State University, U.S.A.); Dr. Y. Valkoun (ICARDA, Aleppo, Syria); Drs. A. Börner and B. Kilian (IPK, Gatersleben, Germany); Drs. O. Raskina, A. Belyayev, E. Nevo (Institute of Evolution, University of Haifa, Israel); Dr. T. Kawahara (Kyoto University, Japan) for providing the material for this investigation. Our work was supported in part by the Russian State Foundation for Basic Research (projects 11-04-00126a, 11-04-00214a, 14-04-00247a) and the Program "Live Nature" from the Presidium of Russian Academy of Sciences.

Open Access This chapter is distributed under the terms of the Creative Commons Attribution Noncommercial License, which permits any noncommercial use, distribution, and reproduction in any medium, provided the original author(s) and source are credited.

\section{References}

Badaeva ED, Badaev NS, Gill BS, Filatenko AA (1994) Intraspecific karyotype divergence in Triticum araraticum (Poaceae). Plant Syst Evol 192:117-145

Belay $\mathrm{G}$ (2000) Univalent frequency in $\mathrm{F}_{1}$ tetraploid wheat hybrids of different germplasm groups. J Genet Breed 54:67-70

Dedkova O, Badaeva E, Amosova A et al (2009) Diversity and the origin of the European population of Triticum dicoccum (Schrank) Schuebl. as revealed by chromosome analysis. Russ J Genet 45:1082-1091 
Dvořák J (1998) Genome analysis in the Triticum-Aegilops alliance. In: Slinkard AE (ed) Proceedings of the 9th international wheat genetics symposium, Printcrafters Inc., Saskatoon, pp 8-11

Friebe B, Gill BS (1994) C-band polymorphism and structural rearrangements detected in common wheat (Triticum aestivum). Euphytica 78:1-5

Friebe B, Jiang J, Raupp WJ et al (1996) Characterization of wheat-alien translocations conferring resistance to diseases and pests: current status. Euphytica 91:59-87

Jiang J, Gill BS (1994) Different species-specific chromosome translocations in Triticum timopheevii and T. turgidum support the diphyletic origin of polyploid wheats. Chromosom Res 2:59-64

Kawahara T (1997) Screening of spontaneous translocations in cultivated emmer wheat. Wheat Inf Serv 85:45-46

Kawahara T, Taketa S (2000) Fixation of translocation 2A-4B infers the monophyletic origin of Ethiopian tetraploid wheat. Theor Appl Genet 101:705-710

Kihara H (1944) Die Entdeckung des DD-Analysators beim Weizen. Agric Hortic Tokio 19:889-890

Kilian B, Ozkan H, Walther A et al (2007) Molecular diversity at 18 loci in 321 wild and 92 domesticate lines reveal no reduction of nucleotide diversity during Triticum monococcum (Einkorn) domestication: implications for the origin of agriculture. Mol Biol Evol 24:2657-2668

Liu CJ, Atkinson MD, Chinoy CN et al (1992) Nonhomoeologous translocations between group 4, 5 and 7 chromosomes within wheat and rye. Theor Appl Genet 83:305-312

Mori N, Kondo Y, Ishii T et al (2009) Genetic diversity and origin of timopheevi wheat inferred by chloroplast DNA fingerprinting. Breed Sci 59:571-578

Naranjo T, Roca A, Goicoecha PG, Giraldez R (1987) Arm homoeology of wheat and rye chromosomes. Genome 29:873-882

Nishikawa K (1984) Species relationship of wheat and its putative ancestors as viewed from isozyme variation. In: Sakamoto S (ed) Proceedings of the 6th international wheat genetics symposium, Plant Germ-Plasm Institute, Kyoto University, Kyoto, pp 59-63

Rodríguez S, Perera E, Maestra B et al (2000) Chromosome structure of Triticum timopheevii relative to T. turgidum. Genome 43:923-930

Salamini F, Ozkan H, Brandolini A et al (2002) Genetics and geography of wild cereal domestication in the Near East. Nat Rev Genet 3:429-441

Salina EA, Leonova IN, Efremova TT, Roder MS (2006) Wheat genome structure: translocations during the course of polyploidization. Funct Integr Genomics 6:71-80

Schlegel R (1996) A compendium of reciprocal translocations in wheat. 2nd edition. Wheat Inf Serv 83:35-46

Schneider A, Molnár I, Molnár-Láng M (2008) Utilisation of Aegilops (goatgrass) species to widen the genetic diversity of cultivated wheat. Euphytica 163:1-19

Taketa S, Kawahara T (1996) C-banding analysis on wild Emmer (Triticum dicoccoides Korn) strains with and without spontaneous reciprocal translocations. Theor Appl Genet 92:173-178 